\title{
Erratum: X-ray powder diffraction data for alaptide, 8(S)-methyl-6,9- diazaspiro/4,5/decane-7,10-dione or cyclo(L-Alanyl-1-ami-no-1-cyclopentan carbonyl), cyclo(L-Ala-Acp) (Volume 24, Issue 1)
}

\author{
J. Maixner ${ }^{\mathrm{a})}$ \\ Central Laboratories, Institute of Chemical Technology Prague, Technická 5, 16628 Prague 6, \\ Czech Republic \\ J. Rohlíček and B. Kratochvíl \\ Department of Solid State Chemistry, Institute of Chemical Technology Prague, Technická 5, \\ 16628 Prague 6, Czech Republic
}

A. Šturc

Interpharma a.s., Komořanská 955, 14300 Prague 4, Czech Republic

(Received 15 May 2009; accepted 26 May 2009)

[DOI: $10.1154 / 1.3154388]$

Below is the corrected Table I originally published on page 33 in March 2009 issue:

TABLE I. Indexed X-ray powder diffraction data for alaptide. Only the peaks with $\mathrm{I}_{\text {rel }}$ of 1 or greater are given. Lattice parameters: $a=21.136(4) \AA, b=7.212(4) \AA, c=6.126(3) \AA, \mathrm{V}=933.8(8) \AA^{3}$ and $Z=4$. All lines were indexed and are consistent with the $\mathrm{P} 2{ }_{1} 2_{1} 2_{1}$ space group.

\begin{tabular}{ccccccccc}
\hline \hline $\begin{array}{c}2 \theta_{\text {obs }} \\
(\mathrm{deg})\end{array}$ & $\begin{array}{c}d_{\text {obs }}, I_{\text {rel }} \\
(\AA)\end{array}$ & $h$ & $k$ & $l$ & $\begin{array}{c}2 \theta_{\text {cal }} \\
(\mathrm{deg})\end{array}$ & $\begin{array}{c}d_{\text {cal }} \\
(\AA)\end{array}$ & $\begin{array}{c}\Delta 2 \theta \\
(\mathrm{deg})\end{array}$ & $\begin{array}{c}2 \theta_{\text {obs }} \\
(\mathrm{deg})\end{array}$ \\
\hline 8.414 & 10.501 & 100 & 2 & 0 & 0 & 8.356 & 10.568 & 0.058 \\
14.909 & 5.937 & 81 & 2 & 1 & 0 & 14.859 & 5.957 & 0.050 \\
16.735 & 5.293 & 1 & 2 & 0 & 1 & 16.714 & 5.300 & 0.021 \\
17.634 & 5.026 & 1 & 3 & 1 & 0 & 17.584 & 5.040 & 0.050 \\
20.875 & 4.252 & 2 & 4 & 1 & 0 & 20.823 & 4.2625 & 0.052 \\
22.222 & 3.9972 & 1 & 4 & 0 & 1 & 22.200 & 4.0012 & 0.022 \\
22.841 & 3.8902 & 2 & 3 & 1 & 1 & 22.831 & 3.8919 & 0.010 \\
24.699 & 3.6016 & 24 & 0 & 2 & 0 & 24.668 & 3.6061 & 0.031 \\
25.609 & 3.4757 & 4 & 5 & 0 & 1 & 25.582 & 3.4793 & 0.027 \\
26.118 & 3.4091 & 24 & 2 & 2 & 0 & 26.089 & 3.4129 & 0.021 \\
27.803 & 3.2062 & 2 & 3 & 2 & 0 & 27.769 & 3.2101 & 0.034 \\
28.227 & 3.1589 & 1 & 6 & 1 & 0 & 28.170 & 3.1653 & 0.057 \\
30.008 & 2.9754 & 2 & 4 & 2 & 0 & 29.976 & 2.9786 & 0.032 \\
31.441 & 2.8430 & 1 & 3 & 2 & 1 & 31.438 & 2.8433 & 0.003 \\
32.655 & 2.7401 & 4 & 5 & 2 & 0 & 32.613 & 2.7435 & 0.042 \\
33.897 & 2.6424 & 1 & 8 & 0 & 0 & 33.902 & 2.6420 & -0.005 \\
35.646 & 2.5167 & 2 & 6 & 2 & 0 & 35.600 & 2.5200 & 0.046 \\
36.162 & 2.4820 & 2 & 8 & 1 & 0 & 36.179 & 2.4808 & -0.017 \\
36.241 & 2.4767 & 1 & 5 & 0 & 2 & 36.187 & 2.4803 & 0.054 \\
37.059 & 2.4239 & 1 & 8 & 0 & 1 & 37.026 & 2.4260 & 0.033 \\
38.909 & 2.3128 & 1 & 6 & 0 & 2 & 38.934 & 2.3114 & -0.025 \\
\hline \hline
\end{tabular}

\footnotetext{
${ }^{a)}$ Author to whom correspondence should be addressed. Electronic mail: Jaroslav.Maixner@vscht.cz
} 